TITRE: LES SUPPORTS NUMÉRIQUES POUR ENSEIGNER, QUELS OBSTACLES ? LITTÉRATIE NUMÉRIQUE SCOLAIRE ET PRATIQUES ENSEIGNANTES

Auteur(s): Georges Ferone, Université Paris-Est, Patricia Richard-Principalli, Université PARIS 8, JACQUES CRINON, UNIVERSITÉ PARIS-EST CRÉTEIL

Publication: Perspectives actuelles Sur L'APprentissage de LA LECTURE ET de L'ÉCRITURE/ CONTRIBUTIONS ABOUT LEARNING TO READ AND WRITE - ACTES DU SYMPOSIUM INTERNATIONAL SUR LA LITÉRACIE À L'ÉCOLE/INTERNATIONAL SYMPOSIUM FOR EDUCATIONAL LITERACY (SILE/ISEL) 2015

PAGES: $364-383$

Directeurs: Marie-France Morin, Denis Alamargot et Carolina Gonçalves.

ÉdiTEUR: LES ÉdITIONS DE L'UNIVERSITÉ DE SHERBROOKE, 2016.

ISBN: 978-2-7622-0355-4

URI: HTTP://HDL.HANDLE.NET/11143/10276

DOI: HTTPS://DOI.ORG/10.17118/11143/10276 


\title{
Les supports numériques pour enseigner, quels obstacles ? Littératie numérique scolaire et pratiques enseignantes
}

\author{
Georges Ferone, Université Paris-Est \\ Patricia Richard-Principalli, Université Paris 8 \\ Jacques Crinon, Université Paris-Est Créteil
}

Résumé : Cette recherche questionne la spécificité des supports numériques à visée éducative et leur usage par des enseignants de l'école primaire. Nous formulons l'hypothèse que l'absence de prise en compte des aspects spécifiques de la littératie numérique scolaire par les enseignants renforce les inégalités d'apprentissage. L'étude a touché trois classes de $5 e$ année en zone d'éducation prioritaire et en école standard. Des séances d'histoire, conduites avec l'appui de documents numériques consultés par les élèves sur un site Internet, ont été filmées et analysées. Les résultats montrent que les activités proposées et l'action des enseignants privilégient la recherche d'informations ponctuelles et l'exploration pas à pas des pages-écrans. En revanche, l'action des enseignants est peu orientée vers une explicitation des objectifs de la leçon et des stratégies cognitives à mettre en œuvre pour construire les connaissances historiques visées. L'utilisation d'un document numérique semble ajouter à la difficulté à organiser la séquence autour de son objectif.

Mots-clés : littératie numérique scolaire, pratiques enseignantes, support d’apprentissage numérique.

\begin{abstract}
This study questions the specificity of digital media for educational purposes and their use by primary school teachers. We hypothesize that teachers' lack of attention to the specific aspects of school digital literacy reinforces learning inequalities. The study involved three Grade 5 classes. History class, conducted with the support of digital documents were filmed and analyzed. At the end of the sequence, we evaluated what they had learned. The results show that the tasks proposed to the students and the action of the teachers foster the search for specific information and the linear exploration of the web pages. The difficulties of computer manipulation also occupies a significant part of the time. Teachers did not gear their action towards explicating the objectives of the lesson and the cognitive strategies to be used to build historical knowledge. Moreover, using a digital document seems to increase the difficulty of keeping the learning objective as a thread of the sequence.
\end{abstract}

Keywords: digital literacy, teaching practices, digital learning support. 
Une des conséquences majeures du développement des technologies numériques est d'accroitre de manière exponentielle les activités de lecture et d'écriture (Jaffré, 2004). Les utilisateurs d'ordinateurs et d'applications numériques en réseau, quels que soient leurs objectifs ou leurs tâches, sont en effet dans l'obligation de lire des textes électroniques (OCDE, 2011). Ces moyens d'information offrent des opportunités pour le lecteur. Ils permettent en effet d'accéder plus rapidement à une quantité plus grande d'informations que les documents sur papier, ce qui peut favoriser une compréhension plus approfondie d'un sujet. Cependant, des travaux mettent en évidence les obstacles à la compréhension que dressent les supports numériques, en particulier chez les lecteurs fragiles (Crinon, 2012; Rouet, 2012). Ces travaux font écho à ceux qui relativisent les compétences des jeunes (Baron et Bruillard, 2008; Fluckiger, 2008) : si l'utilisation des technologies est fréquente chez les adolescents, les usages varient fortement et, pour beaucoup d'entre eux, le spectre d'utilisation est très limité, avec un faible degré de conceptualisation et un degré d'autonomie relatif. Ces observations sont confirmées par les évaluations PISA qui montrent une grande hétérogénéité dans les performances en compréhension des écrits numériques ainsi qu'une forte corrélation entre les performances en compréhension des écrits numériques et celles en compréhension sur support papier (PISA, 2011). Ces résultats réaffirment l'importance de l'école et du rôle des enseignants dans le développement des compétences en littératie numérique pour tous les élèves.

Dans ce contexte, il y a lieu de s'interroger sur les pratiques des enseignants lorsqu'ils font utiliser des documents numériques à leurs élèves. Notre recherche questionne les modes d'approche des documents numériques à l'école primaire au regard des spécificités de la littératie numérique. Notre hypothèse est que l'absence de prise en compte de ces spécificités renforce les inégalités entre élèves dans l'acquisition des compétences en littératie. C'est pourquoi, après avoir défini ce que nous entendons par littératie numérique scolaire, nous analyserons la conduite d'une séquence d'enseignement de l'histoire s'appuyant sur un document numérique, dans trois classes de $5^{e}$ année (cours moyen 2e année) de la région parisienne.

\section{La littératie numérique scolaire}

À partir des concepts de littératie (Barré-De Miniac et al., 2004), de littératie scolaire (Bautier, 2015) et de littératies numériques (Duplàa, 2011), nous proposons d'appeler littératie numérique scolaire les compétences à construire des connaissances et à raisonner, dans le contexte des activités proposées par l'école, avec ou à partir des documents numériques. Certes, on trouve souvent le terme « littératie » employé dans une acception large pour désigner le lire-écrire « dans la diversité de ses usages et dans la diversité de ses modèles théoriques qui permettent de comprendre leur transmission, leur apprentissage et leur mise en œuvre » (Barré-De Miniac et al., 2004, p. 8), ou « l'aptitude à comprendre et à utiliser l'information écrite dans la vie courante, à la maison, au travail et dans la collectivité en vue d'atteindre des buts personnels et d'étendre ses connaissances et ses capacités » (OCDE, 1995); mais nous nous référerons ici plus précisément à ce que nous en apprennent les psychologues, les historiens et les anthropologues sur les liens entre le langage écrit et les modes 
de raisonnement et de pensée. Les genres langagiers, dans la perspective socioculturelle de l'apprentissage et du développement issue des travaux de Vygotski, conduisent à différentes formes de mise en relation des concepts et constituent de puissants transformateurs cognitifs; les formes, les supports et la matérialité même de l'écrit influent sur les manières de penser et de produire les connaissances, de transmettre celles-ci et d'interpréter les textes (Grafton, 2015). Et, parce qu'elles sont socialement et historiquement construites, ces compétences en « littératie » constituent « un ensemble de pratiques » à replacer « dans les contextes singuliers de [leurs] usages sociaux », comme le met en évidence le courant des New Literacy studies (Fraenkel et Mbodj, 2010, p. 10). II n'y a donc pas une littératie abstraite mais des littératies; le contexte scolaire est un contexte spécifique et nous nous proposons de l'étudier en tant que tel. Quelle sont les particularités de la littératie scolaire?

La première de ces particularités est que les supports de l'écrit y sont présents à des fins d'apprentissage-enseignement des différentes disciplines ou domaines du savoir. La littératie scolaire est donc en même temps un moyen et une fin; les genres langagiers sont des outils sémiotiques pour les apprentissages et des objets d'apprentissage. Comme pour d'autres outils sémiotiques, on peut faire l'hypothèse que leur usage et le retour réfléchissant sur cet usage constituent un moteur d'appropriation des compétences, dans une « spirale instrumentale », pour reprendre l'expression utilisée par Alcorta (1998) à propos du brouillon.

Une deuxième particularité concerne les modes de travail intellectuels mobilisés par cette présence des textes et documents à l'école, que Bautier (2015) évoque comme des

« exigences cumulées de raisonnement et de connaissances qui supposent une familiarité avec la fréquentation de l'écrit : mise en relation entre des éléments linguistiques et discursifs qui peuvent être éloignés les uns des autres et les inférences qu’elles supposent [...] ; mobilisation de connaissances scolaires, voire extrascolaires afin de construire l'univers d'interprétation du texte, mais simultanément prise au sérieux du texte comme texte; distinction entre les formes orales et écrites de la langue et de ses usages plus généralement discursifs, mobilisation de processus de secondarisation de la langue et des objets du monde; référence à la notion d'auteur, et plus généralement à celle d'énonciation » (p. 12).

Une troisième particularité tient à la familiarité avec les formes d'écrit et les supports proposés aux élèves, formes et supports qui ont connu une évolution au cours des dernières décennies, qu'il s'agisse des manuels, des albums, des fiches ou de l'introduction des documents numériques (Bautier, Crinon, Delarue-Breton et Marin, 2012; Bonnéry, 2015).

Parler de littératie numérique scolaire revient à s'intéresser, à l'intérieur de ce cadre conceptuel, aux compétences particulières que les élèves développent dans la fréquentation des documents numériques à l'école et dans l'usage qu'ils en font. L'émergence des technologies a conduit les chercheurs à forger de nouveaux concepts pour appréhender la lecture et l'écriture avec le numérique. La terminologie varie avec le temps et les objets de recherche. Goodfellow (2011) recense : computer (-based, 
-assisted, -mediated) literacy, (online-, networked-, electronic-, e-) literacy; Duplàa (2011) évoque les littératies technologiques, médiatiques, littéraTIC; Lebrun, Boutin et Lacelle (2012) parlent de littératie médiatique multimodale.

Les travaux sur les littératies numériques se sont longtemps focalisés sur la recherche d'informations. Aujourd'hui, ils prennent en compte à la fois la dimension de la réception (lecture), de la production (écriture), la diversité des médias utilisés et la communication en réseau. S'il n'existe pas de consensus, la définition de Duplàa (2011) est régulièrement citée : "Les littératies numériques renvoient à la capacité de chercher, de sélectionner, de comprendre, d'interpréter, de produire et de diffuser des informations numériques (écrits, sons et images) sur un réseau en développant des compétences en communication, de définition d'une identité et d'une culture numérique dans l'ensemble des réseaux virtuels » (p. 7). Cependant, à l'école, il ne s'agit pas simplement de chercher, de comprendre et de communiquer de l'information mais bien de répondre aux exigences scolaires, c'est-à-dire de construire des savoirs et de produire et d'échanger en utilisant un langage précis et conforme aux exigences propres des disciplines enseignées. En conséquence, nous préciserons notre définition précédente de la littératie numérique scolaire comme la capacité à chercher des informations, à les utiliser et à produire des significations, en ayant recours à différentes modalités et à différents codes sémiotiques liés au langage écrit, au son et à l'image, et en maitrisant les fonctionnalités des technologies afférentes, pour construire des savoirs propres aux domaines et aux disciplines scolaires et à communiquer selon des exigences langagières spécifiques.

Parler de littératie numérique scolaire implique donc de caractériser les supports numériques dans leurs spécificités. Ceux-ci offrent des particularités par rapport à d'autres supports plus ordinaires dans les pratiques d'enseignement, et exigent la construction de compétences particulières de la part de leurs utilisateurs.

\section{Les spécificités du support numérique}

Les supports numériques font partie des nouveaux supports « composites » que les enseignants utilisent de plus en plus fréquemment, et souvent de manière précoce, dans les classes. Bautier et al. (2012) décrivent les supports composites comme étant: 
- non continus: juxtaposition de modules d'information de taille variable, sans organisation rhétorique évidente, sans liens logiques explicités et sans discours d'ensemble unificateur;

- $\quad$ hétérogènes du point de vue sémiotique : on y trouve notamment, et en relation les uns avec les autres, des textes écrits, des images, des tableaux, des graphiques, des icônes, des légendes;

- hétérogènes du point de vue de l'énonciation (effacement des repères des situations d'énonciation, mélange des attitudes d'énonciation (récit/discours), des registres de l'écrit et de le l'oral, incertitude sur le régime épistémique de ce qui est rapporté (valeurs de vérité), etc.;

- équivoques dans les intentions et les enjeux de communication (instruire, distraire, convaincre, etc.).

À ces caractéristiques partagées avec d'autres supports (albums de littérature de jeunesse, manuels scolaires, etc.) s'ajoutent des caractéristiques propres au numérique. Ainsi, par rapport aux documents composites sur papier, le support numérique ajoute une forte complexité avec l'introduction de nouveaux médias (images animées, sons) et la possibilité de naviguer dans une collection infinie de ressources, grâce à des liens « hypertextuels ». Deux autres caractéristiques sont spécifiques au document numérique : la présentation et les contenus sont en permanence modifiables. Le support physique d'affichage du texte est en effet indépendant du texte lui-même, ce qui modifie sa présentation selon l'appareil utilisé. En outre, les fonctionnalités de l'Internet apparues avec le web 2.0 font coexister des textes non modifiables et d'autres en continuelle évolution. Ces différentes caractéristiques rendent complexes la lecture et la compréhension des supports numériques, ainsi que leur utilisation comme supports d'apprentissage.

\section{Littératie numérique et inégalités scolaires}

Les évaluations nationales et internationales témoignent d'un échec du système scolaire français à réduire les inégalités entre élèves, en particulier en matière de littératie (DEPP, 2015; OCDE, 2011). Rémond (2006) a mis en relation les points de faiblesse des élèves français lors des évaluations internationales avec les pratiques pédagogiques auxquelles sont exposés les élèves de PIRLS, à un âge proche de celui des élèves de notre recherche ${ }^{1}$. Certaines difficultés sont plus accentuées chez les élèves français que dans l'ensemble de l'échantillon : rédiger des réponses construites, se représenter les tâches de lecture, exercer un contrôle métacognitif sur leur lecture, activités qui relèvent de ce que nous avons défini plus haut comme la littératie scolaire. Rémond souligne que ces aspects sont peu enseignés dans l'école française.

1. PIRLS évalue les compétences de lecture des élèves de 49 pays en 4 e année de la scolarité élémentaire. Les enseignants des élèves évalués indiquent, par questionnaire, les tâches qu'ils proposent à leurs élèves. 
Or, il s’agit justement de compétences très différenciatrices socialement. S'emparer des textes de manière fine pour raisonner, prendre du recul sur son activité de lecteur et sur les activités langagières elles-mêmes, tout cela caractérise un rapport « scriptural-scolaire » au langage ou une « secondarisation » (Bautier et Rochex, 2004) en rupture avec ses usages ordinaires. La littératie scolaire met souvent en difficulté les élèves issus de milieux populaires, où sont souvent privilégiés des codes fondés sur les besoins de la communication immédiate, implicite, en situation, quand l'école met en avant prise de distance et élaboration de discours de savoir.

Une partie des difficultés des élèves les plus éloignés des dispositions langagières attendues par l'école vient de ce qu'il leur est difficile de se représenter la nature de l'activité cognitive requise et les enjeux des tâches proposées pour les enseignants. D'où des « malentendus » entre des élèves qui s’acquittent simplement des tâches demandées et des enseignants qui, par ces tâches, visent, souvent sans le dire, la construction de savoirs et de compétences littératiées (Bautier et Goigoux, 2004). Fréquemment, les moyens et les stratégies pour lire et comprendre les textes ne sont pas explicités, les enjeux d'apprentissage des situations proposées sont tus, voire rendus invisibles par l'habillage ludique ${ }^{2}$ de la situation ou l'objectif immédiat de réalisation. On peut penser que ce caractère implicite ou invisible des enjeux, des savoirs sur la langue, les discours et les supports, et des stratégies à mettre en œuvre est un obstacle à la réussite des apprentissages visés (Crinon et al., 2015), notamment en matière de littératie numérique scolaire.

Comme les élèves ne sont pas égaux face aux supports de lecture et d'apprentissage, les pratiques enseignantes constituent des déterminants essentiels de la réussite, notamment des élèves fragiles ou éloignés de la littératie scolaire. L'absence d'explicitation des attendus des tâches d'apprentissage prenant en compte des documents numériques est un obstacle, pensons-nous, à la fois à la réussite des apprentissages notionnels visés et à la compréhension des documents supports.

\section{Méthodologie}

\section{Participants et passations}

Nous avons mené une étude dans trois classes de $5^{e}$ année (élèves de 10-11 ans) d'école primaire en région parisienne (deux en zone prioritaire accueillant une forte proportion d'élèves en difficulté, (classe L et classe R), et une en école standard, que nous appellerons classe J; 43 élèves ${ }^{3}$ ont participé au travail (21 filles, 22 garçons), au cours de l'année 2013-2014. Notre équipe a sélectionné une ressource éducative ${ }^{4}$ correspondant à un point du programme d'histoire qui n'avait pas encore été traité avec les élèves concernés; elle a déterminé un objectif d’apprentissage commun à toutes les classes

2. Cet habillage ludique est presque systématique dans les applications numériques à usage pédagogique.

3. Deux classes étaient à double niveau (CM1-CM2); nous avons travaillé uniquement avec les CM2 (5e année)

4. http://education.francetv.fr/activite-interactive/napoleon-bonaparte-du-consulat-a-l-empire-o13341 
('instauration par Napoléon d'un régime autoritaire) et les évaluations des acquis des élèves en fin de séquence. Les enseignants avaient accepté de conduire une séquence d'apprentissage constituée de deux séances d'une heure environ, incluant le temps de l'évaluation. Ils devaient intégrer à leur séquence l'utilisation par les élèves, sur ordinateur, de la ressource numérique sélectionnée, à l'exclusion d'autres documents. Pour le reste, ils étaient libres de leur démarche pédagogique et du déroulement du travail.

\section{Corpus}

Trois ensembles de données ont donc été analysées:

- le support numérique;

- les productions des élèves;

- les pratiques enseignantes (préparations et séances filmées).

Il s'agit pour nous de mettre en relation étroite les choix et décisions des enseignants, qu'il s'agisse des choix opérés lors de la planification ou lors des interactions en classe, le support d'apprentissage qu'ils utilisent et les productions des élèves aux épreuves d'évaluation.

\section{Méthodologie d'analyse}

Le support. Il est analysé au regard des caractéristiques des supports numériques présentées plus haut.

Les évaluations des élèves. À l'issue de la séquence, les élèves devaient remplir un QCM, constitué de questions factuelles et conceptuelles. En second lieu, les élèves devaient répondre à la question ouverte suivante, directement liée à l'objectif notionnel de la leçon : « Montre en quoi Napoléon se comporte comme un nouveau roi ». Les élèves pouvaient à nouveau se référer au site. Les réponses des élèves ont été analysées en fonction de deux critères: 
- le type de cohérence de l'énoncé produit par l'élève : cohérence causale en lien avec le genre ${ }^{5}$ explicatif historique attendu (voir Deleplace, 2007), cohérence chronologique en lien avec une séquentialité narrative ${ }^{6}$, absence de cohérence causale ou chronologique la cohérence dominante de chaque énoncé d'élève a été caractérisée par le nombre de mots correspondant à chaque catégorie;

- $\quad$ sa pertinence par rapport à la question posée, déterminée par la proportion de mots répondant à la question.

Les pratiques enseignantes. Elles peuvent s'analyser en termes de « registres d'action » (Vinatier, 2007). Vinatier distingue ainsi le registre épistémique, qui concerne la construction des savoirs, le registre relationnel, qui relève de la « dimension intersubjective des échanges » entre l'enseignant et les élèves, et le registre pragmatique, qui engage le pilotage et la régulation de l'action. Nous avons prêté ici une attention particulière à la dimension épistémique. Nous avons essayé de repérer, dans les interactions entre les enseignants et les élèves, celles qui semblaient concerner la littératie numérique scolaire : comment les enseignants présentent-ils à leurs élèves un savoir historique ou étayent-ils la construction de celui-ci, en l'occurrence l'évolution du régime politique de la France sous Napoléon, en s’appuyant sur les apports du support numérique retenu?

En reprenant les caractéristiques de la littératie numérique scolaire, nous analysons les pratiques observées selon trois axes:

1. la prise en compte du support comme outil d'apprentissage de l'histoire,

2. la prise en compte des caractéristiques du support,

3. les modes de travail intellectuel mobilisés.

Pour comprendre les résultats de l'ensemble des élèves, et en particulier ceux des élèves de zone prioritaire, nous présentons les caractéristiques du support numérique utilisé, ses obstacles à la compréhension et les pratiques enseignantes selon la méthodologie proposée.

\section{Une ressource numérique : Napoléon Bonaparte}

Cette ressource numérique a été réalisée par la société Pense-Tête ${ }^{8}$, spécialisée dans la production de multimédia éducatif et culturel, pour le compte de France TV éducation. C'est un « module pédagogique qui propose de découvrir la période napoléonienne du Consulat à l'Empire». Elle comporte

5. Rappelons que dans notre perspective, vygotskienne, le genre relève de «dispositifs de communication socio-historiquement définis» (Maingueneau, 2007, 30) à la fois moyens et objets d'apprentissage, intériorisés et progressivement incorporés, qui permettent de penser et de construire de la signification tant en réception qu'en production.

6. Définie ici comme suite chronologique d'actions et d'évènements.

7. Lorsque les élèves produisent une suite d'informations incohérentes.

8. http://www.pense-tete.com/ 
cinq pages-écrans accessibles par une barre de navigation à la forme de frise chronologique : les liens y apparaissent sous forme de mots (Consulat, Empire), de dates $(1799,1815)$ ou sous les deux formes (Sacre, 1804). Les textes sont contenus dans des cartouches. Trois activités sous différentes formes d'appariement sont proposées au lecteur.

Ce support présente les caractéristiques du composite (Bautier et al., 2012), redoublées par celles propres à tout hypermédia ${ }^{9}$, à savoir les traits conjoints suivants:

- hétérogénéité sémiotique, liée à la pluralité des codes utilisés, tantôt statiques tantôt dynamiques : le support s'appuie sur une frise chronologique et présente une association de textes et d'images de natures variées (dessin d'un personnage coiffé d'un tricorne sur chaque écran, symboles associés à chaque « masse de granit », reproduction du tableau de David, carte de l'Empire, multiples éléments visuels décoratifs ou symboliques : main de justice, branche de laurier, etc.).

- hétérogénéité discursive, liée à l'enchevêtrement des voix, aux situations de double énonciation (plusieurs lecteurs supposés), aux visées différentes : l'un des textes mêle ainsi discours direct et récit sans démarcation visible («Bonjour! Je suis un des premiers lycéens de France! Un Corse du nom de Napoléon Bonaparte est à l'origine des lycées en 1802. Napoléon est né à Ajaccio le 15 août 1759. »); les consignes sont tantôt délimitées par l'italique, tantôt non démarquées du texte explicatif, auquel peuvent également s’adjoindre des indications de navigation; certains éléments utilisés sans explication nécessitent des connaissances historiques expertes et s'adressent à des adultes avertis (par exemple l'expression «masses de granit », extraite d'un discours de Napoléon utilisée comme titre sans guillemets ni contextualisation); la visée des activités ludo-éducatives est ambigüe : sous couvert d'apprentissage, par leur mise en œuvre (il suffit de cliquer au hasard jusqu'à ce que l'on tombe sur la réponse attendue), elles reviennent en réalité à un divertissement dénué d'enjeu de savoir, ce que montrera l'activité des élèves observés.

- discontinuité : le texte, qui relève du récit didactique historique et qui, à ce titre, adopte une structure narrative associée à une logique explicative, est fragmenté en diverses séquences apparemment autonomes, elles-mêmes constituées d'unités micro-textuelles dont la cohérence est parfois à reconstituer (ainsi certaines reprises pronominales n'ont pas de référent: « un tiers LUI est arraché »). Le parcours relaté, depuis le Consulat jusqu'à la chute de l'Empire, depuis un régime intégrant des avancées de la Révolution jusqu'au retour à un pouvoir absolu et sa fin, se subdivise en unités thématiques qui, si elles reproduisent un déroulement chronologique, sont autonomisées par leur organisation spatiale : plusieurs écrans par unité, s’appuyant sur une activité qui illustre ce que dit le texte, de manière souvent peu explicite, et qui nécessiterait une synthèse et l'explicitation des inférences à faire. Or, c'est au lecteur de saisir la macrostructure qui donne sens à l'ensemble des unités juxtaposées, et de comprendre en quoi et comment Napoléon poursuit à la fois l'œuvre de la Révolution et revient vers un régime de pouvoir absolu.

9. Les sites éducatifs proposent essentiellement soit des quizz, soit un équivalent en ligne du récit historique didactique " classique » pour lequel l'interactivité est anecdotique (cf : http://www.de-gaulle-edu.net/degaulle ou http://www. chateau-fontainebleau-education.fr/pages/dossiers/napoleon/napoleon_ home.html. Le site de France Télévisions par la multitude et la diversité des ressources proposées est donc assez unique en son genre, pour le moment. 


\section{Les résultats des élèves}

Faute de place, nous n'indiquerons ici que les principaux résultats ${ }^{10}$. L'analyse des QCM montre que tous les élèves sont capables de chercher des informations ponctuelles (les classes $L$ et $R$ répondent à plus de $90 \%$ aux trois questions factuelles, tout comme la classe J). Par contre, les élèves, en particulier en zone prioritaire, éprouvent des difficultés à répondre à la question qui supposait de sélectionner et de synthétiser les informations éparses sur le site (38\% des élèves de la classe L répondent juste, $8 \%$ de la classe $\mathrm{R}$ et $56 \%$ pour la classe J).

Les élèves des classes en zone prioritaire ont également des difficultés à produire des textes qui s'inscrivent dans le genre attendu (explicatif historique) et qui répondent à la question posée (41\% des textes de la classe $L$ proposent des informations ponctuelles sans lien chronologique ou logique, $83 \%$ pour la classe R; $43 \%$ des textes de la classe L ne répondent pas à la question, $92 \%$ de la classe R). Les caractéristiques du support et la manière dont les enseignants l'ont utilisé, peuvent expliquer en grande partie la faiblesse de ces résultats.

\section{L'analyse de la pratique enseignante}

Nous avons observé si la manière dont les enseignants ont accompagné leurs élèves dans cette séquence pouvait fournir un élément d'explication aux faibles résultats de ceux-ci lors de l'évaluation.

\section{La prise en compte du support comme outil d'apprentissage de l'histoire}

\section{La place incertaine du document}

Le statut toujours discuté de l'informatique à l'école (quasi-discipline traitée à part ou au service d'apprentissages disciplinaires) se retrouve dans l'approche qu'en ont les enseignants. En effet, le support numérique semble être utilisé en tant que tel, non comme moyen mais comme fin. On l'aperçoit dans l'hésitation de l'enseignante de la classe L, présentant l'activité, au début de la séquence, comme intégrant deux domaines habituellement séparés.

Donc d'habitude on fait informatique le mardi et on fait histoire le jeudi. Aujourd'hui on va faire les deux en même temps. On va travailler en histoire avec le support informatique. (Classe L, séance $1,1^{\text {re }} \mathrm{min}$ )

10. Pour une analyse détaillée des productions des élèves, voir Richard-Principalli, Ferone et Crinon, 2016. 


\section{Un support lu dans sa totalité}

Par ailleurs, les enseignants encouragent leurs élèves à lire le document de manière successive et exhaustive. Il s'agirait ainsi de leur permettre de se l'approprier en tant que tel.

Regarde, on te donne des informations. L'intérêt là du site c'est vraiment de lire toutes les informations qu'on nous donne. Ça peut nous aider. (Classe J, séance 1, 12 min)

Il semble que l'exploration du document dans sa totalité est ici considérée par les enseignants comme un préalable à son utilisation pour construire le savoir historique visé. Une difficulté est de s’appuyer ensuite sur les éléments du document susceptibles de contribuer à l'objectif de la leçon, phase qui n'arrive pas.

Le support numérique: une banque d'activités source de «malentendus »

Cette exploration du site passe, dans les trois classes, par une mise en activité des élèves, notamment grâce aux trois jeux « interactifs » présents sur le site. Ceux-ci consistent à relier des mots à leur définition, à associer des personnages ou des pays à une description. L'activité est pensée comme suffisante, sans qu'il soit besoin d'un retour sur les stratégies de lecture et l'usage du support. Si les élèves sont parfois sollicités pour juger de la difficulté des exercices, ils ne sont pas interrogés sur des difficultés rencontrées pour explorer, comprendre ou utiliser le support.

Aussi les élèves ont-ils été invités à effectuer la totalité de ces jeux. Cela correspond à la pédagogie du « faire » que privilégient dans de nombreux domaines disciplinaires les enseignants du primaire (Bautier et Goigoux, 2004) : le contenu de l'activité semble avoir moins d'importance que l'engagement dans la tâche.

Ces exercices à forme ludique font appel à un vocabulaire et à des concepts complexes, mais il est également possible de les réussir sans en comprendre le sens, par essais et erreurs, ce que la grande majorité des élèves a fait. Ces activités consommatrices de temps peuvent en outre générer des « malentendus » sur les enjeux des tâches proposées. Si ces malentendus ne sont pas spécifiques à l'utilisation du numérique, ils apparaissent renforcés par le caractère ludique, censé motiver davantage les élèves, des activités proposées par ce support.

- Qu'est-ce que vous deviez faire un certain moment?

- Des jeux.

- Des jeux, alors des exercices ludiques, sous forme de jeux.

(Classe R, séance 2, 2e min, rappel centré sur les tâches, non sur les objets d'apprentissage) 


\section{Des objectifs d'apprentissage tus}

Les actions de l'enseignant relevant du registre pragmatique (Vinatier, 2007) répondent pour beaucoup à la contrainte temporelle. Une séance de classe est souvent organisée comme la succession d'une mise en activité des élèves et d'un temps de synthèse orale et écrite. C'est bien ce que nous observons dans les trois classes. Or, le temps de mise en activité est prolongé par la gestion matérielle de l'outil (sur l'ensemble de la séquence, 13\% du temps dans la classe L, $20 \%$ dans la classe R et $4 \%$ dans la classe J) et par la complexité du travail à fournir, ne serait-ce que pour lire l'intégralité des éléments du site (puisque nous l'avons vu, il s'agit de lecture exhaustive). Le temps de synthèse est raccourci d'autant et survient en toute fin de séance, il reste donc inabouti. À cela s'ajoute la difficulté à cerner exactement dans quel but on a recours à un site Internet (fin ou moyen ?).

Rien d'étonnant alors que l'objectif d'apprentissage ne soit pas systématiquement présenté. Il est évoqué une fois en début de séquence dans la classe J sans être rappelé par la suite, il n'est jamais mentionné dans la classe $L$ et dans la classe R, il n'est évoqué qu'en toute fin de séance et d'une manière assez peu explicite.

J'aimerais poser une dernière question, est-ce que vous avez réussi à, à comprendre, parce que tout l'enjeu est là, comment Napoléon est passé d'une république à un empire. (Classe $R$, séance $1,51^{\mathrm{e}} \mathrm{min}$ )

\section{La prise en compte des caractéristiques du support}

\section{L'origine et la fonction du support}

Le support n'est pas présenté : d'où vient-il, qui en est à l'origine, à quoi est-il destiné, ces questions ne sont jamais abordées; les informations données, quand il y en a, sont rapides et elliptiques. L'enjeu en est pourtant capital ici, dans la mesure où l'usage critique d'Internet, qui est une compétence littératiée, va de pair avec l'identification des sources et de leur fiabilité.

Je vais vous distribuer une feuille, de la même manière que on l'a fait d'habitude, ça se présente sous la forme d'un rallye. D'accord et on va avancer historiquement, on va garder en tête cette situation de la France à la fin du XVIII' siècle grâce à l'utilisation d'un site internet et des questions que je vous ai préparées. (Classe $R$, séance 1 , 4e min) 


\section{Le support comme hypermédia}

Si les enseignants passent beaucoup de temps à la gestion du matériel (de 4 à $20 \%$ du temps selon les classes) en expliquant et réexpliquant comment se connecter, comment cliquer, etc., en particulier dans les classes de ZEP, le document numérique utilisé est supposé maitrisé d'emblée. L'aide à la navigation est essentiellement une aide ponctuelle au sein de la page où se trouvent les élèves et par rapport à la question à traiter, mais pas dans l'ensemble du site. Aucun enseignant ne présente le support dans sa spécificité numérique : la coexistence d'éléments statiques et dynamiques, la place accordée aux éléments dynamiques et visuels, aux activités ludo-éducatives, la manière d'y naviguer et de s'y retrouver. Au contraire, le support numérique et son utilisation autonome par les élèves semblent aller de soi, rendant inutile une médiation, comme si la familiarité des élèves avec ces supports complexes était acquise.

Faites comme si vous étiez tout seul devant l'ordinateur. (Classe J, séance 1, 14e min)

\section{Le support comme hétérogène}

Il en va de même avec l'hétérogénéité du document. L'hétérogénéité sémiotique est traitée à propos du tableau du Sacre. Les enseignants des trois classes portent une attention particulière à ce tableau et mènent une activité de description d'image sans pour autant en préciser la fonction de propagande. Les autres éléments iconiques, nombreux sur chaque écran, ne sont jamais présentés. II n'est rien dit de leur nature (frise chronologique, cartes, tableau) ni de leur fonction : certains ont une valeur strictement illustrative (les lettrines des titres), d'autres ont à l'inverse une valeur symbolique (l'aigle royal, les feuilles de laurier).

L'hétérogénéité discursive, par exemple la coexistence sur chacun des écrans d'énoncés de statuts et de visées différents, n’est jamais envisagée.

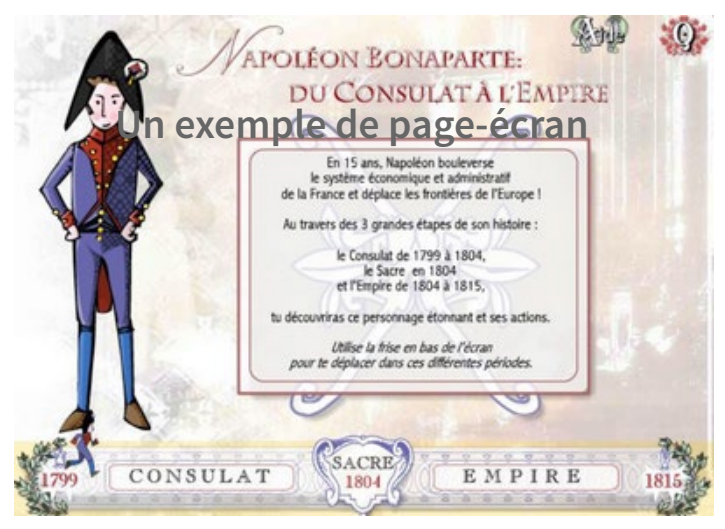


L'écran reproduit ci-dessus présente à la fois des faits sur Napoléon, des informations sur le contenu du site et des instructions de navigation sans distinction de statut mis à part l'italique utilisé pour les consignes de navigation. Le lecteur est interpelé (« tu découvriras ») et le personnage fortement mis en valeur («il déplace les frontières de l'Europe » comme on déplacerait des montagnes, c'est «un personnage étonnant»).

La priorité des enseignants ne semble donc pas de faire construire un parcours parmi de multiples éléments susceptibles de concourir à la signification en fonction de leur pertinence quant à l'objectif de la leçon. Les informations sont traitées successivement, sans être reliées de manière à montrer l'évolution du régime politique.

\section{Les modes de travail intellectuel mobilisés}

Un tissage ${ }^{11}$ davantage centré sur les tâches que sur les objets de savoir

Les trois enseignants contextualisent systématiquement la séance d'apprentissage en procédant à un rappel de la séance précédente, dans le souci de construire un continuum disciplinaire et de mobiliser les prérequis attendus pour poursuivre la séquence. Or, toujours du fait du statut incertain du recours à l'ordinateur, ces rappels sont centrés principalement sur les modalités de travail (utilisation d'Internet) ou sur des informations ponctuelles recueillies sur le site.

La semaine dernière, qui peut me rappeler ce que nous avons fait ensemble? [...] On a travaillé sur Napoléon Bonaparte. Avec quel moyen nous avons travaillé sur Napoléon Bonaparte? [...] Qu'est-ce que tu devais faire, c'était quoi ton travail ? [...] Des exercices, comment est-ce qu'on appelle ça? On en fait régulièrement en informatique. [...] Un rallye. (Rappel centré sur les modalités de travail, Classe L, Séance 2, $1^{\mathrm{re}} \mathrm{min}$ )

On va essayer de... de se rappeler un petit peu pendant cinq minutes ce que l'on a retenu de la... de la séance précédente sur le personnage de Napoléon... qu'avons-nous retenu ? [...] II était capitaine, capitaine de quoi ? (Rappel centré sur des informations ponctuelles, Classe $R$, Séance 2, ge min)

Des savoirs peu hiérarchisés et noyés dans un grand nombre d'informations, à l'image de ce que propose le site

Pour répondre à l'objectif de séance, les élèves devaient comprendre les notions de démocratie et de régime autoritaire. L'enseignante de la classe $L$ aborde la seconde notion lors de la seconde séance. Les élèves lisent le support, puis elle les invite à s'interroger.

11. Au sens où Bucheton et Soulé (2009, p. 8) l'entendent comme «l'activité du maître ou des élèves pour mettre en relation le dehors et le dedans de la classe, la tâche en cours avec celle qui précède ou qui suit, le début avec la fin de la leçon ». 
Et donc on apprend qu'il instaure le régime autoritaire du consulat, quel est le mot qui vous choque, dans régime autoritaire du Consulat? Cloé, y a pas un mot qui te choque? Pourquoi autoritaire? Pourquoi c'est choquant d'avoir ici autoritaire par rapport à ce qui s'est passé avant? (Classe L, Séance 2, $15^{\mathrm{e}} \mathrm{min}$ )

Face à l'incompréhension des élèves, l'enseignante précise le contexte de sa question, elle cite "Louis XIV et sa belle perruque », l'abolition des privilèges, la République, mais elle conclut sans définir ce qu'est un régime autoritaire, ce que le support ne définit pas non plus.

On ne veut plus de l'autorité, mais malgré tout on revient à un régime autoritaire, donc on nous dit que le consulat commence en 1799 et se termine en 1804. (Classe L, Séance 2, $16^{\text {e }} \mathrm{min}$ )

L'enseignant de la classe R évoque également la notion de dictature personnelle en fin de séance. En guise de synthèse de séance, il lit un extrait du site qu'il commente très rapidement, encore une fois en raison du temps qui presse.

Une dictature, c'est un... c'est un régime politique dans lequel tous les pouvoirs appartiennent... sont dans les mains d'une seule personne... c'est-à-dire que Napoléon va s'attribuer... TOus les pouvoirs... c'est lui qui va décider... de TOUT. (Classe R, Séance 2, 35e min)

Or, reproduisant à son insu la démarche du site, qui fourmille de détails d'importance diverse, cet enseignant apporte pour chaque page-écran un très grand nombre d'informations sans les relier à l'objectif de la séance, ce qui permet difficilement aux élèves de distinguer l'essentiel de l'anecdotique.

C'était pour se faire voir, d'accord, aujourd'hui nos... nos souverains à nous quand ils veulent... se montrer, ils organisent une conférence de presse, ils passent à la télé, tout le monde les voit... d'accord ?... Alors que un empereur ou un roi, lorsqu'il doit se montrer et affirmer son autorité vis-à-vis de son peuple, il est obligé de se déplacer... d'accord ? il n’y avait pas la télévision, donc il est obligé de se déplacer... d'accord ?... il appartient en tout cas à la, à la dynastie... d'un roi... il est légitime pour ça. Pourquoi ? parce que justement il a été fait... roi de droit... divin... et que du coup tous ses enfants seront eux-mêmes légitimes dans cette fonction... d'accord? Il est légitime! Par contre, l'empereur n'est pas légitime. (Explications données à propos du tableau du sacre, l'enseignant aborde de manière implicite la notion de propagande, Classe $R$, séance $2,14^{\mathrm{e}} \mathrm{min}$ ) 
Des modes de travail qui privilégient la recherche d'informations ponctuelles

Cette sorte d'effet miroir du site se manifeste aussi dans la démarche que les enseignants mettent en œuvre, ils se laissent ainsi conduire par le site lui-même, faute d'avoir une idée assez claire de ce que peut être l'objectif de travail avec ce support-là. Dans la classe L, l'enseignante pose 30 questions de recherche d'information ponctuelle sur le site, 23 dans la classe J et 11 dans la classe R. À l'inverse, les activités de résumé ou de synthèse des informations sont rares : 4 pour $\mathrm{L}, 3$ pour $\mathrm{J}, 4$ pour $\mathrm{R}$ sur l'ensemble de la séquence. Le rallye proposé dans les classes $L$ et $R$ est constitué de questions, de textes à trous et d'extraits de texte à recopier. L'essentiel de l'activité des élèves consiste donc à retrouver des informations explicites sur le support et à les recopier. De même, les interactions lors de la synthèse se résument le plus souvent à un jeu de questions et réponses pour retrouver les informations ponctuelles présentes sur le support.

Quels sont les métiers de Napoléon à l'âge de 16 ans et de 24 ans ? [...] Alors que fait-il à l'âge de 16 ans ? [...] Qu'est-ce qu'il y a de marqué ? [...] II était? Regarde là. [...] II révèle ses talents militaires mais quel était son métier? [...] Il était capitaine. Très bien. Donc maintenant, ça te permet de remplir ton tableau. (Classe L, séance 1, 23e min)

Ainsi, au final, les interventions des enseignants visent peu la construction d'un savoir historique, savoir qui supposerait une démarche fondée sur la sélection, la synthèse et l'explication. Et, de fait, les évaluations des élèves indiquent qu'ils n'ont pas, pour la plupart, construit ce type de savoir.

\section{Conclusion}

Nous avons défini la littératie numérique scolaire comme la capacité à chercher des informations, à les utiliser et à produire des significations, en ayant recours à différentes modalités et à différents codes sémiotiques liés au langage écrit, au son et à l'image, et en maitrisant les fonctionnalités des technologies afférentes, pour construire des savoirs propres aux domaines et aux disciplines scolaires et à communiquer selon des exigences langagières spécifiques.

Lors de la séquence d'histoire étudiée dans trois classes de CM2, les élèves manifestent des compétences fort diverses à cet égard. Ces compétences semblent pour partie fonction de l'action de leurs enseignants. Ainsi, la recherche et la restitution d'informations ponctuelles constituent ce que les élèves sont les plus nombreux à réussir. C'est aussi ce que les enseignants mettent en avant dans les tâches qu'ils proposent et dans leurs interventions verbales. En revanche, la mise en relation critique de ces informations entre elles, avec des connaissances antérieures ou amenées par l'enseignant et avec les objectifs cognitifs de la leçon n'est opérée que par un petit nombre d'élèves. De fait, ces mises en lien sont peu accompagnées par les enseignants au cours de la séquence et peu verbalisées. 
Il en va donc avec le support numérique comme avec d'autres supports : pour réussir, les élèves doivent manifester des compétences littératiées à utiliser l'écrit pour raisonner et construire des savoirs, mais ces compétences ne leur sont pas explicitement enseignées (Rochex et Crinon, 2011). Les modes de travail intellectuel décrits ici présentent les mêmes limites que ceux pointées par Rémond (2006) à propos des activités proposées en lecture compréhension; il n'y a pas ou peu d'activité d'explicitation et d'argumentation (Britt et Rouet, 2012), d'activité analytique et synthétique (Coirier, Gaonac'h et Passerault, 1996) et les malentendus mis par exemple en évidence par Bautier et Goigoux (2004) sont renforcés par le caractère pseudo-ludique du support.

Enfin, les caractéristiques spécifiques du support, l'hétérogénéité sémiotique, l'hétérogénéité discursive et la discontinuité ne sont pas prises en compte par les enseignants, et d'ailleurs ne semblent pas perçues. La perception des obstacles semble se concentrer sur les problèmes techniques de prise en main de la machine ou de fonctionnement de celle-ci. Ces difficultés réelles ajoutent sans doute encore aux contraintes, en réduisant le temps disponible pour des interventions centrées sur les savoirs.

Le support induit même un oubli de l'objectif de la leçon : il n'y a pas d'objectif indiqué dans le site, conçu comme un outil librement utilisable par les enseignants; or les manuels, même lorsqu'ils sont composites, mentionnent toujours les objectifs d'apprentissage. Le support numérique rend ainsi la tâche plus difficile pour les enseignants puisqu'il les oblige à faire eux-mêmes ce que les manuels leur donnent «clé en main ». La conséquence que nous avons observée ici est que les enseignants multiplient les éléments d'information, les uns anecdotiques les autres essentiels, et qu'ils ne les ressaisissent pas en un discours explicatif visible et articulé logiquement; en cela, ils miment les apports du site, qui présente la même discontinuité (apport d'informations par cumulation, hiérarchisation invisible, macro-structure transparente) et qui requiert des opérations cognitives particulièrement complexes de la part des élèves pour qu'ils puissent lire, construire la compréhension des informations écrites proposées et apprendre.

Loin d'aller de soi, comme le donnent à entendre les discours sur la génération des digital natives et les prescriptions institutionnelles, l'usage des ressources numériques s'apprend. Sans cela, il pourrait constituer un nouvel agent des inégalités scolaires. C'est pourquoi la littératie numérique scolaire constitue à nos yeux une priorité dans la formation des enseignants. 


\section{Bibliographie}

Alcorta, M. (1998). Une approche vygotskienne du développement des capacités d'écrit : le brouillon, un outil pour écrire? Dans M. Brossard et J. Fijalkow (dir.), Apprendre à l'école, perspectives piagétiennes et vygotskiennes (p. 123-151). Talence : Presses universitaires de Bordeaux.

Baron, G.-L. et Bruillard, E. (2008). Technologies de l'information et de la communication et indigènes numériques : quelle situation? Revue des Sciences et Technologies de l'Information et de la Communication pour l'Education et la Formation, STICEF [en ligne], 15 [consulté le 23 novembre 2016]. Disponible sur le Web : http://sticef.univ-lemans.fr/num/vol2008/09r-baron/sticef_2008_baron_09.htm

Barré-De Miniac, C., Brissaud, C. et Rispail, M. (dir.) (2004). La littéracie. Conceptions théoriques et pratiques d'enseignement de la lecture-écriture. Paris : L'Harmattan.

Bautier, É., Crinon, J., Delarue-Breton, C. et Marin, B. (2012). Les textes composites : des exigences de travail peu enseignées? Repères, 45, 63-79.

Bautier, É. (2015). Quand la complexité des supports fait obstacle à la compréhension de tous les élèves. Spirale, 55, 11-20.

Bautier, É. et Goigoux, R. (2004). Difficultés d'apprentissage, processus de secondarisation et pratiques enseignantes: une hypothèse relationnelle. Revue française de Pédagogie, 148, 89-100.

Bautier, É. Rochex, J.-Y. (2004). Activité conjointe ne signifie pas significations partagées. Dans C. Moro et R. Richenmann (dir.), Situation éducative et significations (p. 197-220). Bruxelles : De Boeck.

Bonnéry, S. (dir.) (2015). Supports pédagogiques et inégalités scolaires. Paris: La Dispute.

Britt, M. A. et Rouet, J.-F. (2012). Learning with multiple documents: Component skills and their acquisition. Dans M.J. Lawson \& J.R. Kirby (dir.), The Quality of Learning: Dispositions, Instruction, and Mental Structures. Cambridge University Press, 276-314.

Bucheton, D. et Soulé, Y. (2009). Les gestes professionnels et le jeu des postures de l'enseignant dans la classe : un multi-agenda de préoccupations enchâssées. Éducation et didactique, 3(3), 29-48.

Coirier, P., Gaonac’h, D. et Passerault, J.-M. (1996). Psycholinguistique textuelle. Paris : Armand Colin.

Crinon, J. (2012). Enseigner le numérique, enseigner avec le numérique. Le français aujourd'hui, 178, 107-114.

Crinon, J. (2011). Lire à l'ère numérique. Argos, 48, 20-24.

Crinon, J., Espinosa, N., Gremmo, M.-J., Jarlégan, A., Kreza, M. et Leclaire-Halté, A. (2015). Clarté cognitive et apprentissage du lire-écrire au CP: quelles pratiques enseignantes? Pratiques, 165-166.

Deleplace, M. (2007). Le récit comme accès à la connaissance historique. Réflexions didactiques sur le récit historique. Pratiques, 133-134, 33-53. 
DEPP. (2015). Journée Défense et Citoyenneté 2014 : un jeune sur dix handicapé par ses difficultés en lecture. Note d'information - DEPP, $n^{\circ} 16$. Paris : Ministère de l'Éducation nationale.

Duplàa, E. (2011). Lire et écrire internet : définition, enjeux et évaluation des littératies numériques. Dans A. Desrochers et M.-J. Berger (dir.), L'Évaluation de la littératie (p. 255-286). Ottawa : Presses de l'Université d'Ottawa.

Fluckiger, C. (2008). La culture numérique des élèves est-elle une culture comme les autres ? Revue Française de Pédagogie, 63, 51-61.

Fraenkel, B. et Mbodj, A. (2010). Introduction. Les new literacy studies, jalons historiques et perspectives actuelles. Langage et société, 133, 7-24.

Goodfellow, R. (2011). Literacy, literacies, and the digital in higher education. Teaching in Higher Education, 16(1), 131-144.

Grafton, A. (2015). La page, de l'antiquité à l'ère du numérique. Paris : Hazan.

Jaffré, J.-P. (2004). La litéracie : histoire d'un mot, effets d'un concept. Dans C. Barré-De Miniac, C. Brissaud et M. Rispail (dir.), La littéracie. Conceptions théoriques et pratiques d'enseignement de la lecture-écriture (p. 21-41). Paris : L’Harmattan.

Lebrun, M., Boutin, J.-F. et Lacelle, N. (2012). La littératie médiatique multimodale : de nouvelles approches en lecture-écriture à l'école et hors de l'école. Québec: PUQ.

Maingueneau, D. (2007). Genres de discours et modes de généricité. Le français aujourd'hui, 159, 2935.

OCDE. (1995). Littératie, économie et société. Résultat de la première enquête sur l'alphabétisation des adultes. Paris: OCDE.

OCDE. (2011). Résultats du PISA 2009. Élèves en ligne: Technologies numériques et performance (Volume VI). En ligne : http://dx.doi.org/10.1787/9789264113015-fr

Richard-Principalli, P., Ferone, G. et Crinon, J. (2016). Support numérique et écriture de textes de savoir au cycle 3 de l'école primaire. Communication au colloque «Enseignement et apprentissage de l'écriture de la maternelle à l'université et dans les formations tout au long de la vie », Bordeaux, 19-21 octobre 2016.

Rémond, M. (2006). Éclairages des évaluations internationales PIRLS et PISA sur les élèves français. Revue française de pédagogie, 157, 71-84.

Rochex, J.-Y. et Crinon, J. (éds.). (2011). La construction des inégalités scolaires. Rennes : Presses Universitaires de Rennes

Rouet, J.-F. (2012). Ce que l'usage d'internet nous apprend sur la lecture et son apprentissage. Le français aujourd'hui, 178, 55-64.

Vinatier, I. (2007). La notion d'organisateur dans une perspective interactionniste. Recherche et formation, 56, 33-46. 